ESJ Social Sciences

\title{
Relation Proche Aidant/ Aidé Et Accompagnement Familial : Regard Sur La Maltraitance Des Personnes Agées Dépendantes En Perte D'autonomie (Côte d'Ivoire)
}

\author{
Ahou Clémentine Tanoh Say \\ Département de Sociologie, Université Félix Houphouët-Boigny ((UFHB), \\ Laboratoire d'Etude et de Recherche Interdisciplinaires en Sciences Sociales \\ (LERISS) Abidjan, Côte d'Ivoire
}

Doi:10.19044/esj.2021.v17n5p200

Submitted: 23 November 2020

Accepted: 12 February 2021

Published: 28 February 2021
Copyright 2021 Author(s)

Under Creative Commons BY-NC-ND

4.0 OPEN ACCESS

Cite As:

Tanoh Say A.C. (2021). Relation Proche Aidant/Aidé Et Accompagnement Familial : Regard Sur La Maltraitance Des Personnes Agées Dépendantes En Perte D’autonomie (Côte d'Ivoire). European Scientific Journal, ESJ, 17(5), 200.

https://doi.org/10.19044/esj.2021.v17n5p200

\section{Résumé}

En Côte d'Ivoire, la maltraitance envers les personnes âgées dépendantes en perte d'autonomie par leurs proches aidants, reste encore silencieuse, non prévue ni punie par la loi. Et pourtant, elle existe réellement dans les différents domiciles où les aînés passent leur fin de vie. C'est pour analyser cette réalité sociale que la présente étude a été menée du 12 avril au 15 septembre 2020, avec 15 aînés et 15 aidants familiaux affiliés à l'ONG "Âge d'Or" située géographiquement dans la commune de Cocody. A l'issue de l'analyse de contenu thématique des données qualitatives recueillies par des entretiens individuels et discussions de groupes, les caractéristiques sociales des proches aidants, leurs perceptions des aînés et de leurs rôles ont été déterminées. Les formes de maltraitance que subissent les aidés de la part des proches aidants ont été identifiées et l'impact de la maltraitance sur les aînés a été analysé. L'épuisement lié à la durée de l'aide dans le temps et à la pléthore des tâches à accomplir quotidiennement, couplé aux conditions socioéconomiques, relationnelles et familiales qui existent entre les aidés et les aidants explique cette maltraitance. Pour mieux apprécier l'ampleur de ce problème social, l'étude doit être élargie aux aînés des autres communes. 
Mots clés : Proche aidant, maltraitance, personne âgée, dépendante en perte d'autonomie, Côte d'Ivoire

\title{
Helping / Helped Close Relationship and Family Support: Focus on Abuse of Dependent Elderly in Loss of Autonomy (Ivory Coast)
}

\section{Ahou Clémentine Tanoh Say}

Département de Sociologie, Université Félix Houphouët-Boigny ((UFHB), Laboratoire d'Etude et de Recherche Interdisciplinaires en Sciences Sociales (LERISS) Abidjan, Côte d'Ivoire

\begin{abstract}
In Côte d'Ivoire, mistreatment of dependent elderly people with loss of autonomy by their caregivers is still silent, neither foreseen nor punished by law. And yet, it really exists in the various homes where the elderly spend their end of life. It is in order to analyze this social reality that the present study was conducted from April 12 to September 15, 2020, with 15 seniors and 15 family caregivers affiliated with the NGO " Golden Age ", geographically located in the commune of Cocody. Following the thematic content analysis of the qualitative data collected through individual interviews and group discussions, the social characteristics of the caregivers, their perceptions of the elderly and their roles were determined. The forms of abuse suffered by the carers from the caregivers were identified and the impact of abuse on the elders was analyzed. Burnout related to the duration of the assistance over time and the plethora of tasks to be accomplished on a daily basis, coupled with the socioeconomic, relational and family conditions that exist between carers and helpers explains this abuse. In order to better appreciate the extent of this social problem, the study must be extended to seniors in other municipalities.
\end{abstract}

Keywords: Close caregiver, mistreatment, elderly person, dependent with loss of autonomy, Ivory Coast

\section{Introduction}

En Côte d'Ivoire, le vieillissement rapide de la population n'est pas sans effet sur les familles et soulève de nombreux défis à relever au plan macro social (institutionnel), méso social (régional, communautaire, associatif) et micro social (familial, relationnel). Aussi, la dynamique du nombre des personnes âgées de 60 et plus se constate de plus en plus. De 604934 (soit 
3,4\% de la population totale recensée) en 1998 ( INS $\left.^{1}, 2014\right)$, la proportion des personnes âgées de 60 ans et plus est passée à 913.668 en $2014\left(\mathrm{RGPH}^{2}\right.$, 2014), soit 4.4\% de la population totale évaluée à 22671331 habitants. Cependant, l'insuffisance de politiques d'accompagnement intégrant les besoins des personnes vieillissantes au plan sanitaire, économique, social et juridique s'observe. Or, le vieillissement ne se déroule pas toujours de façon normale, mais s'accompagne très souvent d'une transition épidémiologique c'est-à-dire d'une augmentation de la prévalence des maladies chroniques, des démences et des troubles neurocognitifs $\left(\mathrm{OMS}^{3}, 2016\right)$. Une telle situation peut ainsi induire la dépendance et la perte d'autonomie chez les sujets âgés. Ces problèmes de santé, qui nécessitent une prise en charge plus longue, complexe et coordonnée (OMS, 2013), exigent la mobilisation des ressources humaines, matérielles et financières de la famille, du moins, en Côte d'Ivoire. En effet, ce pays ne disposant pas encore d'établissements spécialisés de soins de longue durée pouvant accueillir les personnes âgées dépendantes en perte d'autonomie, la seule alternative reste l'entourage immédiat ou la famille où un soutien informel peut leur être apporté par des proches aidants. Le terme proche aidant ou aidant familial ou aidant naturel désigne « toute personne de l'entourage qui apporte un soutien significatif, continu ou occasionnel, à titre non professionnel, à une personne ayant une incapacité. Il peut s'agir d'un membre de la famille ou d'un ami » (MSSS, cité dans Carrier et al., 2017). Tel que défini, le proche aidant se présente comme un acteur indispensable du processus d'accompagnement des personnes vieillissantes dépendantes et en perte d'autonomie. La dépendance est « l'impossibilité partielle ou totale, pour une personne, d'effectuer sans aide les activités de la vie, qu'elles soient physiques, psychiques ou sociales, et de s'adapter à son environnement » (Vallée, 2017). En Côte d'Ivoire, le nombre de "personnes dépendantes" était de 189371 (47 246 hommes et 142125 femmes) en 1998 (INS, 2014). Cette sous-population représentait $31,3 \%$ des personnes de grand âge. L'autonomie est définie par la capacité à se gouverner soi-même. Elle présuppose la capacité de jugement, c'est-à-dire la capacité de prévoir et de choisir, et la liberté de pouvoir agir, accepter ou refuser dans le respect des lois et des usages communs (Henrard, 2012). "Les mots dépendance et autonomie renvoient aux modes de relations entre les individus dans notre société » (Ennuyer, 2013). Ici, ces modes de relation concernent les personnes âgées et leurs proches aidants. Globalement, ce sont les proches aidants qui procurent de l'aide au profit des aînés. Les rôles joués par ceux-ci sont parfois complexes et variés, pouvant aller du partenariat dans les soins de santé primaire (Carrier et al., 2017) jusqu'à la gestion des biens matériels et financiers, passant par

\footnotetext{
${ }^{1}$ Institut National de la Statistique

${ }^{2}$ Recensement Général de la Population et de l'Habitat.

${ }^{3}$ Organisation Mondiale de la Santé
} 
l'aide à la satisfaction des besoins fondamentaux de l'aîné, selon les constats empiriques. Assumer de telles responsabilités implique certaines aptitudes telles que la disponibilité, l'endurance, la compréhension et la patience chez les aidants. Or, ces derniers n'ont pas été formés à cela. Avec le temps, la lassitude apparaît et la tolérance de l'aidante s'épuise pour faire place à la maltraitance (Lestrade, 2014). « Il y a maltraitance « quand un geste singulier ou répétitif, ou une absence d'action appropriée, se produit dans une relation où il y devrait avoir de la confiance, et que cela cause du tort ou de la détresse chez une personne aînée. Elle peut prendre différentes formes (maltraitance physique, maltraitance psychologique, maltraitance financière...) »(OMS, 2015). La présente étude s'inscrit dans la construction théorique autour des causes du mauvais traitement familial envers les personnes âgées. Cette théorie a été influencée par le modèle situationnel qui suggère que les circonstances qui entourent l'individu victime d'une situation d'abus sont extrêmement importantes (Bennett et al. cités dans Ibañez, 2013). L'une de ces circonstances serait «le stress expérimenté par la famille dans un certain contexte, de sorte que, au fur et à mesure que le stress de l'agresseur augmente, augmente aussi la probabilité de commettre des actes abusifs dirigés contre un individu vulnérable, considéré comme source de la situation de stress » (Ibañez, 2013). En Côte d'Ivoire, les données sur ce sujet sont quasi inexistantes. Le fait que les aînés demeurent et évoluent au sein de leurs familles respectives pourrait justifier cette relative insuffisance de données scientifiques sur cette question. La présente étude se veut donc une contribution à documenter cette réalité. Elle questionne la relation de maltraitance des proches aidants envers leurs aînés dépendants en perte d'autonomie et vise à l'analyser.

\section{Methodologie}

\subsection{Site et population}

La présente étude s'est déroulée à Cocody, l'une des communes de la ville d'Abidjan. Avec une superficie de $132 \mathrm{~km}^{2}$, la commune de Cocody enregistre 447055 habitants (INS, 2014) parmi lesquelles des personnes âgées dépendantes en perte d'autonomie qui sont assistées d'aidants familiaux dans leur existence quotidienne. Ces deux catégories sociales constituent l'objet de la présente étude. Deux raisons fondamentales expliquent le choix de cette commune : la commune de Cocody regorge de nombreux immeubles et villas de haut standing qui meublent ses belles cités faisant ainsi sa renommée. Toutefois, l'architecture de ces immeubles est souvent inappropriée pour les personnes âgées dépendantes qui éprouvent des difficultés pour y accéder par les marches. Ensuite, Cocody bénéficient d'infrastructures et ONG parmi lesquelles l'ONG "Age d'Or" qui œuvre pour la solidarité autour de la personne âgée. 


\subsection{Echantillonnage et échantillon}

La technique d'échantillonnage par choix raisonné a été privilégiée dans cette étude. Cet échantillonnage non probabiliste a permis de sélectionner des participants considérés comme typiques de la population cible. C'est un échantillonnage orienté, ciblé qui a consisté à choisir les éléments de la population sur la base de critères particuliers, afin que les éléments soient représentatifs du phénomène étudié. Ainsi, les participants de l'étude ont été pris sur le listing de l'ONG "Age d'or “. Ils ont été choisis sur la base de critères particuliers suivants :

Pour les aînés : être âgé de 60 ans ou plus, être dépendant en perte d'autonomie, être habitant de la commune de Cocody et être affilié à l'ONG "Âge d'Or".

Pour les proches aidants : être en aide à un parent âgé dépendant en perte d'autonomie depuis plus d'un an, être inscrit sur la liste de l'ONG “Âge d'Or " comme aidant familial et répondant de l'aidé. A partir de ces critères inclusifs, un échantillon composé de 15 personnes âgées dépendantes en perte d'autonomie et de 15 proches aidants, (soit un total de 30 personnes) a été constitué.

\subsection{Technique et processus de collecte de données}

La présente étude s'inscrit dans l'approche qualitative. La principale technique de collecte de données a été l'entretien individuel semi-directif. Toutefois, dans les domiciles, la technique d'observation a aussi été d'une très grande utilité. L'étude s'est déroulée sur une période discontinue de cinq mois, allant du 12 avril au 15 septembre 2020, tenant compte de la disponibilité des proches aidants et de l'état de santé des aidés. Les aînés ont été identifiés le mercredi 6 janvier 2016 au cours du lancement des activités de l'ONG "Âge d'Or '. Au fil des ans, plusieurs d'entre eux sont devenus dépendants à la suite de diverses maladies invalidantes (accident vasculaire cérébral (AVC), maladie d'Alzheimer, dépression mentale, ...) et se font représenter par leurs aidants auprès de cette ONG. Ils sont au nombre de 15 qui n'arrivent plus à se déplacer et habitent la commune de Cocody. Les aidants ont accepté volontairement de participer à l'étude. Selon un calendrier établi, les visites à leurs domiciles respectifs ont permis de rencontrer aussi les aidés et d'obtenir leur accord de principe. Au préalable, il a fallu expliquer aux enquêtés l'utilité d'une telle étude et obtenir leur consentement verbal. Quinze (15) entretiens individuels qui ont duré de 37 à 50 minutes chacun se sont déroulés avec les aînés à leurs domiciles. Avec les aidants familiaux, quinze (15) entretiens individuels et cinq (5) discussions de groupe d'une durée de 90 minutes environ se sont déroulés au siège de l'ONG, après les différentes séances de formation destinées aux aidants familiaux. Ceux-ci ont été rassurés de la discrétion dans laquelle l'étude est menée et de l'anonymat de leurs réponses. 
Ces différents entretiens ont été enregistrés à l'aide d'un dictaphone numérique (IC recorder Sony ICD-P520). Les questions abordées concernent les thématiques suivantes :

Avec les aînés : Leurs caractéristiques sociales ; les formes de maltraitance qu'ils subissent de la part des proches aidants ; l'impact de ces actes de maltraitance sur eux.

Avec les proches aidants : leurs caractéristiques sociales ; leurs perceptions des aînés et des rôles qu'ils jouent.

\subsection{Technique d'analyse des données}

Dans cette approche qualitative, le dépouillement manuel a été retenu comme procédé pour l'analyse thématique des guides d'entretien. Les entretiens enregistrés ont fait l'objet de retranscription et 30 fiches ont été constituées. Ces fiches élaborées ont fait l'objet d'une analyse transversale et les entretiens ont été regroupés par thématique. Une fois ce travail achevé, l'analyse de contenu thématique (Paillé et Mucchielli, 2012) a été retenue comme technique et les catégories analytiques construites. Les unités de signification et les idées maîtresses ont été dégagées.

\section{Resultats}

Les points clés ci-dessous structurent les résultats de cette étude :

- les caractéristiques sociales des proches aidants, leurs perceptions des aînés et de leurs rôles ;

- les caractéristiques sociales des aidés, les formes de maltraitance que subissent les aidés de la part des proches aidants ;

- l'impact de la maltraitance sur les aînés.

\subsection{Caractéristiques sociales des proches aidants, perceptions de leurs â̂nés et de leurs rôles}

\subsubsection{Caractéristiques sociales des proches aidants}

Sur 15 aidants interviewés $(n=15), 13$ sont de sexe féminin et 2 de sexe masculin. Leur âge varie de 32 à 57 ans. Ils sont analphabètes $(\mathrm{n}=5)$ ou déscolarisés $(n=10)$. Ils sont soit sans emploi $(n=10)$, soit femme au foyer $(n=5)$. Elles sont mariées $(n=5)$, divorcée $(n=1)$ ou séparée $(n=1)$, célibataires $(n=6)$, veuves $(n=2)$, ayant tous des enfants à charge. Ils sont enfants biologiques $(n=4)$, conjointes $(n=5)$, neveu ou nièce $(n=2)$, cousines $(n=2)$ ou belles filles $(\mathrm{n}=2)$. L'expérience à la relation d'aide est de 2 ans $(\mathrm{n}=5), 3$ ans $(n=7)$ et 4 ans $(n=3)$. 


\subsubsection{Perceptions des proches aidants de leurs aînés}

Les personnes âgées considérées autrefois comme des références sociales et culturelles, devenues dépendantes en perte d'autonomie à cause des pathologies sénescentes qui les affectent, sont aujourd'hui victimes de stéréotypes généralement négatifs. Ces stéréotypes sont partagés par l'ensemble des aidants familiaux interrogés lors de cette étude. C'est du moins, ce qui ressort de leurs différents discours dont quelques extraits se présentent comme suit :

«Madame, tout se paie sur la terre. Cet homme couché à la maison là est mon oncle. Il était un gradé de l'armée ivoirienne. Il était une fierté pour notre région. Il a aidé beaucoup de gens, mais il a refusé de m'aider à entrer dans l'armée, moi son neveu. Un vrai mystique, un sorcier fin! Aujourd'hui où il n'a plus rien, où il fait tout sur lui, c'est à moi la famille a demandé de venir essuyer son derrière, de venir le soulever. Sa maladie là n'est pas simple. Il est en train de payer devant le bon Dieu tout le mal qu'il m'a fait. » (G.A., 42 ans, proche aidant, célibataire, sans emploi) «Au moment où il m'abandonnait avec les enfants pour courir les petites filles partout, il ne savait pas que ce jour allait arriver. Maintenant, je ne peux plus bouger de la maison, sans qu'il crie mon nom comme un enfant. C'est Dieu même qui l'a terrassé pour me venger avec mes enfants pour tout le mal qu'il nous a fait. Il n'a qu'à appeler ses copines qui ont disparu pour venir nettoyer aussi son "caca" et son "pipi", moi je vais me reposer un peu. Espèce d'adultère, de mari méchant, de père inconscient! »

(E.E. Epouse et aidante familiale, 51 ans).

Les incontinences fécale et urinaire couplées à la dépendance et à la perte d'autonomie modifient le regard de l'entourage envers ces personnes âgées. En effet, à l'analyse des discours, ces dernières ne bénéficient plus de soutien affectif, ni de compassion de la part de leurs différents aidants. A contrario, ces derniers s'inscrivent dans une logique de vengeance et de dépréciation de leurs aidés en rapport à une relation sociale rétrospective jugée insatisfaisante. Ce modèle déprécié des aidés se construit à partir des items «mystique, sorcier fin, adultère, mari méchant et père inconscient » qui constituent des indicateurs de relation de domination de l'aidant à l'aidé.

\subsubsection{Perceptions de leurs rôles par les aidants}

Les rôles joués par les aidants sont multiples et variés. Dans cette relation de l'aidant à l'aidé, l'éducation reçue liée au respect des aînés, les 
qualités et valeurs de l'aidant, les sentiments et émotions éprouvés par celuici, participent de la réussite de cette mission. Comme rôles, les aidants familiaux interrogés ont égrené les tâches journalières qu'ils traduisent en ces mots :

«Je suis tellement submergée par les activités que je ne sais par où commencer. C'est moi seule qui fait tout, vous comprenez, tout, tout! Là, je suis en train de craquer. Je n'en peux plus. " (D.A. nièce et aidante familiale, 39 ans). «Je lave ses habits, je la lave, je la nettoie quand elle a tout fait sur elle, je l'accompagne à l'hôpital, je lui donne ses médicaments, je nettoie sa chambre, je prépare ses repas, je fais ces retraits, je paie ses factures. Elle est devenue comme un bébé pour qui je fais tout. » (K.P. fille et aidante familiale, 37ans).

Selon les discours des aidants, leurs rôles ne se limitent pas seulement à aider les aînés à satisfaire leurs besoins physiologiques (le bain, la toilette en cas de besoin, la prise des repas et des médicaments...). Mais leurs rôles s'étendent aussi aux tâches domestiques telles que le ménage et le rangement dans la chambre à coucher de l'aidé, l'entretien de ses vêtements et draps de lit, la confection de ses repas en fonction de son régime alimentaire. A ces tâches s'ajoutent diverses autres courses comme les veilles de nuit, les visites à l'hôpital, le paiement des factures, les retraits bancaires, l'accueil des visiteurs, etc. Face à cette polyvalence et hyperactivité, les aidants perçoivent leurs rôles conséquemment :

« Je me sens exploitée, abusée, humiliée même. Je suis la bonne à tout faire. Ma vie est devenue comme une sorte de routine qui s'est installée. Et cela fait trois ans que je n'ai plus mis pieds dans une église, ni même dans un salon de coiffure. Je ne peux pas m'épanouir comme les filles de mon âge. Je faisais une formation professionnelle. Mais j'ai tout arrêté depuis trois ans pour m'occuper de maman, car je suis sa seule fille. Hum !» (G.A. fille et aidante familiale, 33 ans) «Mes journées et mes nuits sont très épuisantes. Je ne dors pratiquement plus les nuits depuis quatre ans où mon mari a fait sa crise d'AVC. A force de le soulever, j'ai failli mourir pour le laisser. J'ai des courbatures partout. Je n'ai pas le choix, je suis obligée de le faire. Je ne me suis pas du tout attendue à une si grande épreuve! »(G.T. épouse et aidante familiale de 57 ans). 
Que ce soit dans le premier comme dans le second cas, l'angoisse, la détresse et l'épuisement transparaissent dans les discours des proches aidantes (fille biologique et épouse). Ici, le caractère obligatoire ou involontaire, contraignant et inattendu des rôles d'aidantes émerge des discours. Ces aidantes jouent leurs différents rôles dans un esprit d'humiliation, de sacrifice, de devoir moral, de résignation, de reconnaissance et même de compassion. En bref, ces proches aidantes considèrent leurs rôles auprès des personnes âgées dépendantes en perte d'autonomie comme un lourd fardeau ou de véritables charges très pesantes qui entravent leur liberté.

\subsection{Caractéristiques sociales des aînés et les formes de maltraitance 2.2.1. Caractéristiques sociales des aînés}

Les caractéristiques sociales des aînés objets de cette étude se déclinent comme suit : les deux sexes sont représentés dans l'étude, soit huit (8) hommes et sept (7) femmes. Leur âge varie entre 75 et 89 ans. Ils ont tous le niveau d'étude universitaire et sont issus de diverses catégories socio professionnelles, notamment, des cadres de l'administration ivoirienne $(\mathrm{n}=9)$ ou du secteur privé $(n=6)$. Ils sont mariés $(n=5)$, veufs $(6)$, divorcés $(n=2)$ ou séparés de corps $(n=2)$. Leur expérience à la dépendance et à la perte d'autonomie est de 2 ans $(n=7), 3$ ans $(n=5)$ et 4 ans $(n=3)$. Au niveau sanitaire, ils souffrent d'hémiplégie ou de paralysie totale des suites d'Accident Vasculaire Cérébral (AVC) $(\mathrm{n}=7)$, de dépression et délire accompagnés d'incontinences fécale et urinaire $(\mathrm{n}=3)$, de la maladie d'Alzheimer débutante $(\mathrm{n}=2)$, de cancer au stade terminal $(\mathrm{n}=2)$, du diabète, d'hypertension artérielle et de la cécité $(\mathrm{n}=1)$. En somme, au niveau des aidés, l'on note une prédominance du sexe masculin. Aussi, le quatrième âge (75 ans ou plus) s'affiche-t-il avec ses maladies gériatriques dégénératives, faisant ainsi des aînés, des personnes dépendantes qui ont perdu leur autonomie.

\subsubsection{Les formes de maltraitance envers les aînés par leurs aidants}

Au regard de l'analyse du discours des ainés, l'on constate qu'ils sont victimes de différentes formes de maltraitance selon la classification de l'OMS (2015). Il s'agit notamment des violences physiques, morales et psychologiques, médicamenteuses et financières, des négligences actives et des violations des droits civiques. Les locutions ci-après l'illustrent bien :

«Mon neveu qui s'occupe de moi dit que je suis sorcier, c'est pour cela que je fais tout sur moi. Quand ça le prend, il me laisse tomber dans la douche où il est seul avec moi. Il dit qu'il est fatigué de me soulever, de me nettoyer et qu'à cause de moi, il a mal au dos. Il me dit souvent de mourir pour qu'il ait la paix. Il me fait beaucoup de vilaines choses alors que je le paye à hauteur de $60000 \mathrm{~F} / \mathrm{mois}$. C'était sa condition pour qu'il 
accepte de venir s'occuper de moi. Et comme si cela ne suffisait pas, il me vole beaucoup d'argent. Je le vois mais je ne peux pas parler de peur qu'il ne m'abandonne. Eh! Dieu, hum! pleures... » (E.Y.V. 78 ans, atteint de cancer depuis 4 ans)

Ce septuagénaire dépendant et en perte d'autonomie est perçu négativement par son aidant. Cette perception négative détermine le traitement qu'il subit. IL dit être victime de violences physiques (son neveu le laisse volontairement tomber souvent), psychologiques (il le traite de sorcier, l'accuse d'être à la base de son mal de dos et réclame sa mort) et financières (vol d'argent et exigence de compensation financière en contre partie du service rendu). Une autre aidée renchérit en ceci :

«Ma nièce passe tout son temps à dormir. Quand je l'appelle, elle me répond en ceci : "aaah! Y' a quoi encore? Moi, je suis fatiguée. Pourquoi toi-même, tes enfants ne viennent pas faire tout ça là même? "Donc, souvent les heures de prises des médicaments ne sont pas respectées. Si par exemple, à midi, elle est en train de dormir, je suis obligée de l'attendre et on saute souvent les prises. Les nuits, n'en parlons pas. Quand je l'appelle, elle ne me répond pas du tout. Et je suis obligée de faire "pipi" et "caca" dans ma couche. Avec ça, comment je peux guérir?» (K.A., 75 ans, paralysie totale à la suite de plusieurs crises d'AVC).

Comme relaté dans son discours, cette septuagénaire dit être victime de violences psychologiques (intimidations et réprimandes envers l'aidée), médicamenteuses (inobservance du traitement), de négligences actives et passives (l'indifférence de l'aidante à répondre promptement à ses appels).

\subsection{Impacts de la maltraitance sur les aînés}

Comme définie plus haut, "la maltraitance des personnes âgées entraîne des blessures ou une détresse morale pour la personne âgée qui en est victime »(OMS, 2015). En effet, les violences commises à l'égard des personnes âgées dépendantes en perte d'autonomie provoquent assez de détresse morale et des blessures physiques chez ces dernières. Ces récits le témoignent :

"C'est ma belle-fille qui s'occupe de moi, parce qu'aucune de mes propres filles n'a accepté de me recevoir chez elle, sous prétexte que je suis sorcière. C'est mon unique fils qui a accepté de m'accueillir chez lui. Mais sa femme n'est pas du tout contente de me voir. Donc, vraiment je la remercie beaucoup pour tout. Mais, fréquemment, volontairement, elle 
me laisse tomber. Après, elle dit à mon fils que je suis lourde, donc elle ne fait pas exprès. J'ai eu déjà plusieurs fractures et blessures. Je ferme ma bouche, car si je parle de tout ce que je subis à mon fils, qu'est ce qui peut se passer? Je préfère me taire et souffrir seule dans mon âme et dans mon corps. Hummmm! »(Y.K., 77 ans, dépressive et incontinente urinaire et fécale).

Cette aînée est victime de maltraitance psychologique, d'abandon moral et physique de la part de ses filles biologiques. Elle étale ses souffrances morales, psychologiques et physiques vécues dans sa relation à sa belle-fille, une relation d'aide censée être celle de confiance. Dans ce cas d'espèce, les incontinences fécale et urinaire créent une surcharge de travail chez la proche aidante et peuvent entraîner la maltraitance de l'aidée. Celle-ci se plaint de rejet, d'isolement, voire d'abandon. Un autre octogénaire abonde dans le même sens. Il s'exprime en ces termes :

"Depuis que j'ai perdu la vue, à cause du diabète et de l'hypertension artérielle, c'est mon fils qui fait mes courses. A ma grande surprise, il a vidé tout mon compte et a vendu mon terrain sans mon consentement. Ma douleur est à son comble. Ce garçon me fait terriblement souffrir... »(K. B., 83 ans, diabétique, hypertendu et non voyant).

La détresse morale de cet aidé se constate dans son discours. La maltraitance de ces personnes aînées est favorisée par leur vulnérabilité. Elles sont, en effet, fragilisées par la poly pathologie et le poly handicap. Les aidants familiaux qui les accompagnent dans leur processus de vieillissement ont parfois du mal à supporter ces faiblesses et cette situation. Ce sont de véritables facteurs de risques qui sont liés au profil des aidés qui ne peuvent plus accomplir seuls les actes de la vie quotidienne.

\section{Discussion des Resultats}

De l'analyse des résultats obtenus, émergent trois grands points qui sont : la situation socio-économique et psychologique des proches aidants, la nature de la relation proche aidant/ aidé, les perceptions et réactions des aînés aux comportements maltraitants.

\section{La situation socioéconomique et psychologique des proches aidants}

Les données recueillies indiquent une prédominance du sexe féminin au niveau des aidants familiaux, soit 13 femmes contre 2 hommes sur 15 enquêtés. Ces résultats confirment le rôle traditionnel de la femme africaine ou ivoirienne qui est chargée de s'occuper des travaux ménagers, de tous les membres de la famille et singulièrement des personnes âgées qui meurent 
maternés et sécurisés par les femmes (Golaz, 2015). En effet, ce sont les femmes qui assurent le maintien à domicile de leurs ascendants (Golaz, 2015). Ainsi, les personnes âgées doivent compter sur la présence et le soutien de leurs filles pour faire face à l'accroissement de leur dépendance (AttiasDonfut, 2014). En outre, l'âge des aidants familiaux varie de 32 à 57 ans. Ces données concernant l'âge renseignent que ces aidants sont tous des adultes qui devraient avoir eux-mêmes leurs propres familles. Or, en dehors des cinq (5) épouses qui sont devenues aidantes de leurs conjoints respectifs, les autres sont soit célibataires (6), soit veuves (2), soit divorcée (1) ou séparée (1). Ces dix (10) aidants ont tous des enfants à charge. Leur niveau d'étude (5 analphabètes, 10 déscolarisés) et leur situation socio professionnelle (10 chômeurs et 5 femmes au foyer) pourraient expliquer partiellement leur dépendance matérielle et financière. Hormis les cinq épouses aidantes de leurs maris et le neveu aidant qui perçoit $60000 \mathrm{~F}$ mensuellement, les neuf autres aidants (enfants, nièce, cousine, ...) évoluent dans l'indigence économique. Ils ne perçoivent aucune prime d'encouragement pouvant leur permettre de faire face à leurs propres besoins et à ceux de leurs enfants. En effet, les familles n'intègrent pas toujours systématiquement les besoins des proches aidants alors qu'ils constituent un soutien primordial à la personne âgée dépendante en perte d'autonomie. Dans un tel contexte, les proches aidants sont comme livrés à eux-mêmes face à leurs propres besoins, au stress et à la surcharge des activités à mener quotidiennement. Une telle situation peut induire aussi des cas de maltraitance envers les ainés. Cette maltraitance qui peut se décliner souvent sous forme de vengeance peut être aussi liée à l'augmentation du stress.

\section{La nature de la relation proche aidant/ aidé}

La relation d'aide initialement désintéressée qui perdure pour plusieurs aidants qui comptabilisent deux à quatre années de service se mue progressivement en une relation conflictuelle. A cela s'ajoute le manque de temps de repos pour ces aidants pour qui, l'univers domestique se confond à la sphère du travail alors que pour la majorité, le travail constitue une sphère distincte de celle de la famille (Bricka, 2016). L'enchevêtrement de ces différentes sphères de vie ne peut que contribuer à l'épuisement des aidants familiaux au plan physique, psychologique, émotionnel, social et financier.

Ainsi, l'aide apportée est souvent une source d'épuisement autant physique que psychologique pour l'aidant familial (Ferrero, 2017). En effet, les aidants familiaux interviewés ont mentionné de nombreuses difficultés notamment le manque de temps, les problèmes financiers, le stress, les difficultés physiques et psychologiques. Cette augmentation de stress ne peut qu'augmenter aussi la probabilité de commettre des actes abusifs dirigés contre les aînés dépendants considérés comme la source de la situation de 
stress, selon le modèle théorique situationnel évoqué par Bennett et al. (cités dans Ibañez, 2013). En effet, les difficultés du proche aidant peuvent être reliées au stade de la maladie chez l'aidé, ce dernier impliquant différentes tâches et responsabilités et différents degrés d'implication (Sardas et al., 2017). Les quinze (15) aidants familiaux interrogés ont avoué maltraiter leurs différents aidants en raison de l'épuisement physique, moral, psychologique et financier. Certains ont confié ne plus avoir de souffle pour continuer cette aide. Cet « essoufflement » physique et moral épuise leur tolérance et conduit à la violence envers leurs parents âgés dépendants en perte d'autonomie. Dans cette relation proche aidant/ aidé, il peut arriver que le comportement de l'aidé atteigne un tel niveau répétitif ou démesuré qu'il épuise la tolérance de l'aidant (Lestrade, 2014). La violence familiale à l'égard des personnes âgées est donc souvent liée aux traits du caractère et aux attitudes et comportements de ces dernières. C'est alors que l'aidant souhaite la mort de l'aidé pour qu'il soit enfin libéré. Dans la présente étude, les épouses dominées d'hier s'érigent en maltraitantes comme par vengeance ou par un renversement de pouvoir. Du fait de son état de dépendance, le conjoint dominateur dépend désormais de l'épouse qui était auparavant dominée, et qui devient souvent son soignant principal. C'est aussi le cas des enfants biologiques et des autres membres de la famille (neveu, nièce, cousine...) maltraités et silencieux qui, devenus adultes, deviennent, à leur tour, maltraitants envers leurs parents affaiblis physiquement, intellectuellement et économiquement. Ainsi, les adultes maltraitant leurs parents, ont souvent été eux-mêmes des enfants maltraités. Cet état de fait a été mentionné par un aidant familial qui a reconnu avoir maltraité son oncle qui ' $n$ 'avait rien fait antérieurement pour l'aider à entrer dans l'armée ivoirienne“. La maltraitance d'enfants et de vieillards s'inscrit ainsi dans un cycle intergénérationnel de violences, les maltraitants ayant été eux-mêmes victimes de violences dans leur enfance. La violence devient ainsi un mode de vie habituel dans ces familles, violence contre tous les êtres faibles de la famille : enfants, femmes, et personnes âgées. Toutefois, la maltraitance envers les personnes âgées reste encore assez secrète alors qu'elle continue de se développer en famille. Face à cette situation, il est important d'orienter la réflexion sur la perception et les réactions des aidés aux comportements maltraitants.

\section{Les perceptions et réactions des aînés aux comportements maltraitants}

Les aidés victimes de maltraitance de la part de leurs aidants familiaux ont choisi de garder le silence. Plusieurs raisons peuvent expliquer le nonsignalement des cas de maltraitance.

D'abord, en Côte d'Ivoire, les campagnes de sensibilisation contre les violences sont celles qui concernent les femmes et les enfants. Alors que les personnes âgées sont autant vulnérables que ces deux catégories de personnes. 
Contrairement aux pays développés, il n'existe pas pour l'heure de disposition législative spécifique prévue pour punir les cas de violences faites aux aînés. Dans un tel contexte, le non-dévoilement de la maltraitance par les aînés euxmêmes ou par une tierce personne peut se justifier. En effet, dévoiler un cas de maltraitance sans que les maltraitants ne soient punis par faute de disposition législatives conséquentes ne peut qu'aggraver la situation précaire des aînés et les exposer davantage à plus de maltraitance. Ensuite, l'aidé peut ignorer volontairement la situation de maltraitance vécue pour préserver les liens familiaux (lien conjugal entre le fils et la belle-fille aidante par exemple). L'aîné peut ainsi nier la situation vécue ou, tout simplement, ne pas considérer que les comportements commis sont abusifs, notamment en raison de son bagage familial ou culturel (OMS, 2020). La crainte de ne pas être cru, les réactions de la famille, les conséquences pour le conjoint violent, les croyances religieuses et le désir de préserver l'équilibre familial sont autant de barrières mentionnées aussi par Beaulaurier et al., (2008). Par ailleurs, les aidés, du fait de la dépendance, sont déjà dans un état de vulnérabilité qui ne leur permet pas de réagir contre leurs agresseurs. C'est ce qu'a exprimé cette aidée par «Je préfère me taire et souffrir seule dans mon âme et dans mon corps ». Alors que selon les déclarations de certains, les actes de maltraitance leur causent assez de désagrément, douleur, chagrin, humiliation, honte, indignation ou colère, déshonneur et révolte. Ils se sentent infantilisés par leurs aidants et atteints dans leur intégrité, dignité et autonomie citoyenne (OMS, 2015). Somme toute, ce sont des pleurs qui sont exprimés comme réactions par les aînés enquêtés qui souhaiteraient mourir afin d'être enfin délivrés et de libérer ceux pour qui ils sont devenus une charge.

\section{Conclusion}

En Côte d'Ivoire, la maltraitance envers les aînés dépendants en perte d'autonomie par leurs prochesaidants est un phénomène réel et fréquent. Toutefois, elle demeure encore silencieuse. Elle n'est pas prévue et punie par la loi alors qu'il s'agit d'un problème lourd de conséquences de par les blessures qu'elle occasionne au niveau psychique, physique et en termes de mortalité (OMS, 2020). C'est dans le but de contribuer à documenter ce sujet que cette étude a été initiée dans la commune de Cocody qui regorge de nombreuses personnes âgées retraitées. Parmi celles-ci figurent des personnes dépendantes en perte d'autonomie. L'étude a eu pour objectif d'analyser la relation de maltraitance des proches aidants envers leurs aînés dépendants en perte d'autonomie. A l'issue de l'analyse de contenu thématique des données, les caractéristiques sociales des proches aidants, leurs perceptions des aînés et de leurs rôles ont été déterminées. De même, les formes de maltraitance que subissent les aidés de la part des proches aidants ont été identifiées et l'impact de la maltraitance sur les ainés a été analysé. Comme portée scientifique et 
sociale, cette étude a permis de mettre en exergue un phénomène social insuffisamment documenté en Côte d'Ivoire : celui de la maltraitance des personnes âgées dépendantes en perte d'autonomie. En effet, contrairement au discours social qui laisse admettre que les aînés sont systématiquement intégrés, maternés et sécurisés dans leurs différentes familles jusqu'à leur fin de vie terrestre, l'étude a montré que ces derniers subissent diverses formes de maltraitance. En perspective, cette étude peut être approfondie et élargie à l'ensemble des aînés des autres communes du District Autonome d'Abidjan en vue de mieux apprécier l'ampleur de ce phénomène social. Ainsi, la limitation de l'étude à seulement une quinzaine d'aînés en constitue la limite majeure. Aussi, les études à venir pourront-elles être orientées vers les relations plus privilégiées entre les aidants et leurs aidés afin de déterminer s'il existe aussi des cas de maltraitance dans ce contexte.

\section{References :}

1. Attias-Donfut, C., (2014). Grand-âge, maladie, handicap : le rôle central des aidants. L'école des parents, numéro spécial, (609), 14-16.

2. Beaulaurier, R. L., Seff, L. R., et Newman, F. L., (2008). Barriers to Help-Seeking for Older Women Who Experience Intimate Partner Violence: A descriptive Model. Journal of Woman and Aging, 20 (34), p. 231-248.

3. Bricka, B., (2016). Des vies (presque) ordinaires : paroles d'aidants. Ivry-sur-Seine, France : Les Editions de l'Atelier.

4. Carrier, D. S., Morin, P., Gross, O., De La Tribonniere, X., (2017). L'engagement de la personne dans les soins de santé et services sociaux: Regards croisés France-Québec, Québec : Presses de l'Université du Québec, - 304 pages.

5. Ennuyer, B., (2013). Les malentendus de l' «autonomie» et de la « dépendance » dans le champ de la vieillesse, Dans Le sociographe 15 (Hors-série 6), pages 139 à 157. Les malentendus de la dépendance : de l'incapacité au lien social. Paris, Dunod.

6. Ferrero, M., (2017). La relation aidant-aidé et ses avatars. Le journal des psychologues, (345), 66-70.

7. Golaz, V., Rutaremwa, G., Wandera, S. O., (2015). Les solidarités familiales autour des personnes âgées en Ouganda. Repéré à https:// www.researchgate.net > publication > 280595446_Les_so...

8. Henrard J-C., (2012). La perte d'autonomie : Un nouvel enjeu de société - L'Harmattan, - 145 pages.

9. Ibañez, J. G., (2013). La violence familiale envers les personnes âgées en Espagne Dans Déviance et Société 2013/1 (Vol. 37),pages 67 à 88. Institut National de la Statistique, (2014). Ministère d'Etat, Ministère 
de Plan et du Développement ... Enquête Démographique et de Santé de Côte d'Ivoire. Récupéré à partir de http://www.ins.ci.

10. Lestrade, C., (2014). Les limites des aidants familiaux. Dans Empan ( $\left.{ }^{\circ} 94\right)$, pages 31 à 35 . Repéré à

11. https://doi.org/10.3917/empa.094.0031

12. Organisation Mondiale de la Santé, (2013). Maladies non transmissibles.

Repéré à http://www.who.int/mediacentre/factsheets/fs355/fr/

13. Organisation Mondiale de la Santé, (2015). Maltraitance des personnes âgées. Repéré à http://www.who.int/mediacentre/factsheets/fs357/fr/

14. Organisation Mondiale de la Santé, (2016). Rapport mondial sur le vieillissement et la santé. apps.who.int $>$ iris > bitstream > 9789240694842_fre.

Repéré à https://

15. Organisation Mondiale de la Santé, (2020). Maltraitance des personnes âgées. Repéré à http://www.who.int> ... > Principaux repères > Detail.

16. Paillé, P. et Mucchielli, A., (2012). L'analyse qualitative en sciences humaines et sociales, Quatrième édition, Armand Colin.

17. Recensement Général de la Population et de L'habitat, (2014). « Résultats Abrégés du Recensement Général de la Population et de l'Habitation ", INS, Abidjan. 54 p. Récupéré à partir de http://www.ins.ci.

18. Vallée, A., (2017). La dépendance des personnes âgées : une réflexion éthique, Editions L'Harmattan, - 142 pages 